\title{
Self-Regulation and Other Learning Dimensions in Hybrid Courses: Which Characteristics Matter in Academic Performance?
}

\author{
Claudia Danelia chamorro-Urroz ${ }^{1}$, Jorge Alberto Mosqueda-Benavides ${ }^{2}$, Jorge Membrillo- \\ Hernandez ${ }^{3}$ \\ ${ }^{1,2}$ Digital Education Department, ${ }^{3}$ School of Engineering, Writing Lab Tec Labs. \\ Tecnologico de Monterrey, Ave. Eugenio Garza Sada 2501, 64849 Monterrey, NL, Mexico
}

\begin{abstract}
Hybrid education has been referred to as the 'third generation' of distance education. An increasing number of universities are including hybrid courses within their academic offer, especially due to the worldwide pandemic that COVID-19 has caused. Therefore, it is very important to understand how students deal with the hybrid experience. Previous studies have been focused mainly in $100 \%$ online courses, but little research has been done in hybrid education. It is important to understand how self-regulation and student learning profiles can affect in a positive or in a negative way their academic performance during the course. The data was collected on hybrid courses offered by the Tecnologico de Monterrey in Mexico, through the CEVEAPEU questionnaire to 4,857 students of different careers programs of the 26 different campuses around the country. An exploratory data analysis was performed, as well as a statistical correlation analysis of Pearson and Spearman. Our findings show that the factors that affect the final academic performance of a student more significantly, although with a weak correlation, are intrinsic/extrinsic motivation, anxiety, selfregulation, and information processing. Therefore, we can conclude that, although self-regulation is a dimension that affects performance in hybrid courses, it is not the only dimension, nor it is a determining factor in the final grade on whether a student will pass or fail a course.
\end{abstract}

Keywords: Hybrid Education, Learning Dimensions, Academic Performance, Higher Education, Educational Innovation 